\title{
The Carbon content in the Galactic CygnusX/DR21 star forming region
}

\author{
H. Jakob ${ }^{1}$, R. Simon ${ }^{1}$, C. Kramer ${ }^{1}$, B. Mookerjea ${ }^{1}$, N. Schneider ${ }^{2}$, \\ S. Bontemps ${ }^{2}$ and J. Stutzki ${ }^{1}$ \\ 1 KOSMA, I.Physikalisches Institut, Universitaet zu Koeln, Germany \\ 2 Observatoire de Bordeaux, France
}

\section{Introduction}

Observations of Carbon bearing species are among the most important diagnostic probes of ongoing star formation. $\mathrm{CO}$ is a surrogate for $\mathrm{H}_{2}$ and is found in the vicinity of star formation sites. There, [CI] emission is thought to outline the dense molecular cores and extend into the lower density regions, where the impinging interstellar UV radiation field plays a critical role for the dissociation and ionization processes. Emission of ionized carbon ([CII]) is found to be even more extended than $[\mathrm{CI}]$ and is linking up with the ionized medium. These different tracers emphasize the importance of multiwavelength studies to draw a coherent picture of the processes driving and driven by high mass star formation. Until now, large scale surveys were only done with low resolution, such as the COBE full sky survey, or were biased to a few selected bright sources (e.g. Yamamoto et al. 2001, Schneider et al. 2003). A broader basis of unbiased, high-resolution observations of [CI], $\mathrm{CO}$, and $[\mathrm{CII}]$ may play a key role to probe the material processed by $\mathrm{UV}$ radiation.

We here present a $2 \mathrm{deg}^{2}$ large-scale map of ${ }^{13} \mathrm{CO} 2-1$ (see Figure 1) in the Cygnus X region and follow-up $\left(12^{\prime} \times 14^{\prime}\right)$ maps of the DR21 region in the two [CI] fine structure lines and the ${ }^{12} \mathrm{CO}$ 7-6 rotational transition. Additional KOSMA observations of ${ }^{12} \mathrm{CO} 6-5,{ }^{13} \mathrm{CO} 6-5$, and ${ }^{12} \mathrm{CO} 3-2$ are included for comparison. We compare parts of the KOSMA data with a [CII] map taken with $\mathrm{KAO}$ in $1994,{ }^{12} \mathrm{CO} 9-8$, and ${ }^{13} \mathrm{CO} 9-8$ observations (Boreiko \& Betz 1991, also KAO) and with publicly available ISO-LWS spectra at 7 distinct positions in the vicinity of DR21.

\section{The Observations: CI vs. CO}

The DR21 HII region/molecular cloud core has long been subject of detailed studies, which have led to a comprehensive view of the region. Measuring several parsecs in diameter at a distance of $1.5 \mathrm{kpc}$ (Bontemps et al., in prep.), it harbors probably one of the most extended bipolar outflow in the Galaxy. The region contains a compact HII region at the center and two extended lobes of shocked $\mathrm{H}_{2}$ emission running along an axis from $\mathrm{NE}$ to 
SW. The molecular material found in the lobes was presumably ripped off a dense molecular ridge that is aligned $\mathrm{N}-\mathrm{S}$, and the wind originating from DR21 is about to break through the cavity (Lane et al. 1990).

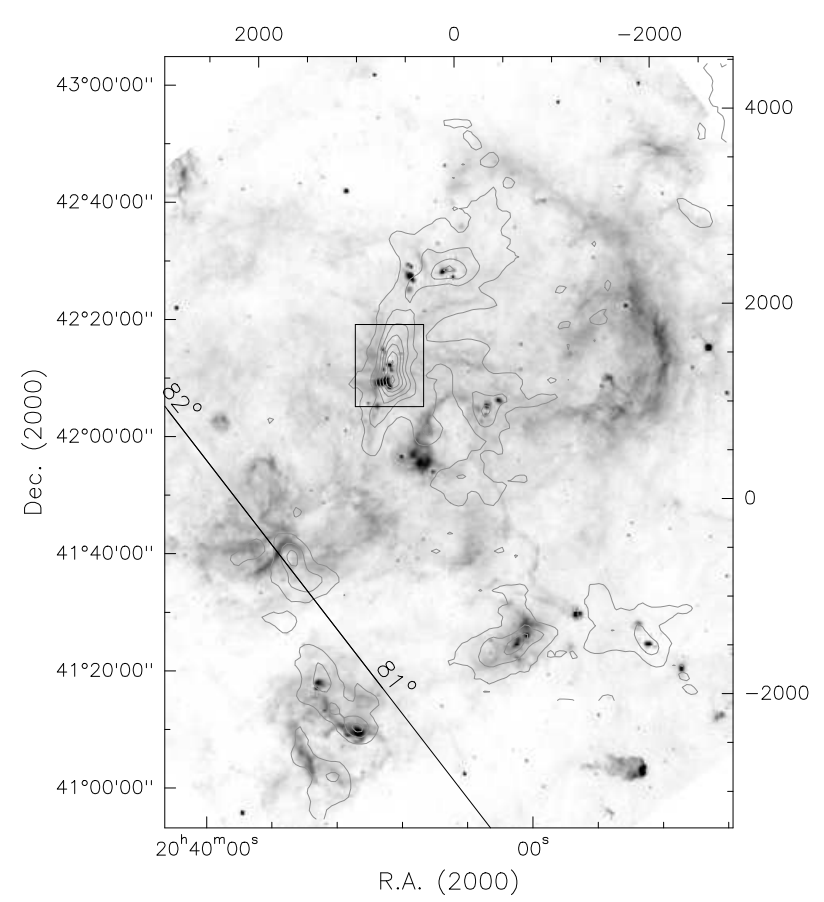

Figure 1:

KOSMA $\quad{ }^{13} \mathrm{CO}$ 2-1 velocity integrated intensity between -6 and 1 $\mathrm{kms}^{-1}$ (contours) on top of an 8 $\mu \mathrm{m}$ MSX image. The DR21 region mapped in both CI lines and $\mathrm{CO}$ 7-6 is marked by a box (cf. Figure 2).

Besides ${ }^{13}$ CO 2-1 (Figure 1), we have gathered observational data of both [CI] lines that are assumed to be optically thin. We find, that the ratio of the integrated intensity is almost constant at about 0.9 in the mapped region (compare with Figure 2). This indicates that the [CI] lines have their origin in a warm environment. The emission roughly follows the shape of the low$\mathrm{J}{ }^{13} \mathrm{CO}$ maps. This clearly is in contrast to the CO 7-6 map, which shows strong emission toward the DR21 core and the outflow lobes, but not along the ridge in the vicinity of DR21(OH) and FIR1 (cf. Figure 3).

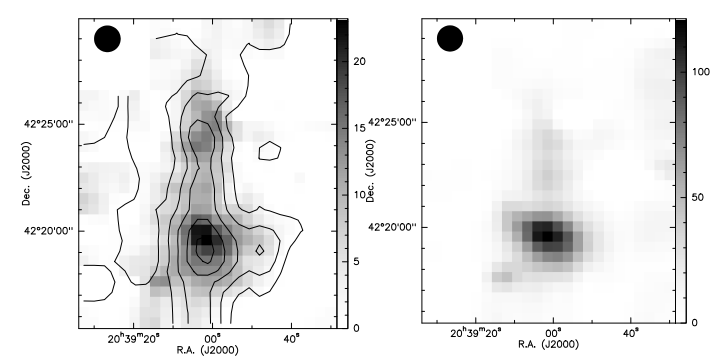

Figure 2: $\quad$ KOSMA observations of [CI] ${ }^{3} \mathrm{P}_{1}-{ }^{3} \mathrm{P}_{0}$ (left, in contours), [CI] ${ }^{3} \mathrm{P}_{1}-{ }^{3} \mathrm{P}_{0}$ (greyscale) and $\mathrm{CO}$ 7-6 (right) of the DR21/DR21(OH) region. 


\section{The ISO LWS sample}

Seven ISO-LWS positions (see Figure 3) cover the core (\#2), the east and west lobe of the DR21 outflow (\#1 and \#3) and two positions in between (DR21 East and West). The two nearby sources DR21(OH) and DR21 FIR1 lie inside the dust lane to the north.

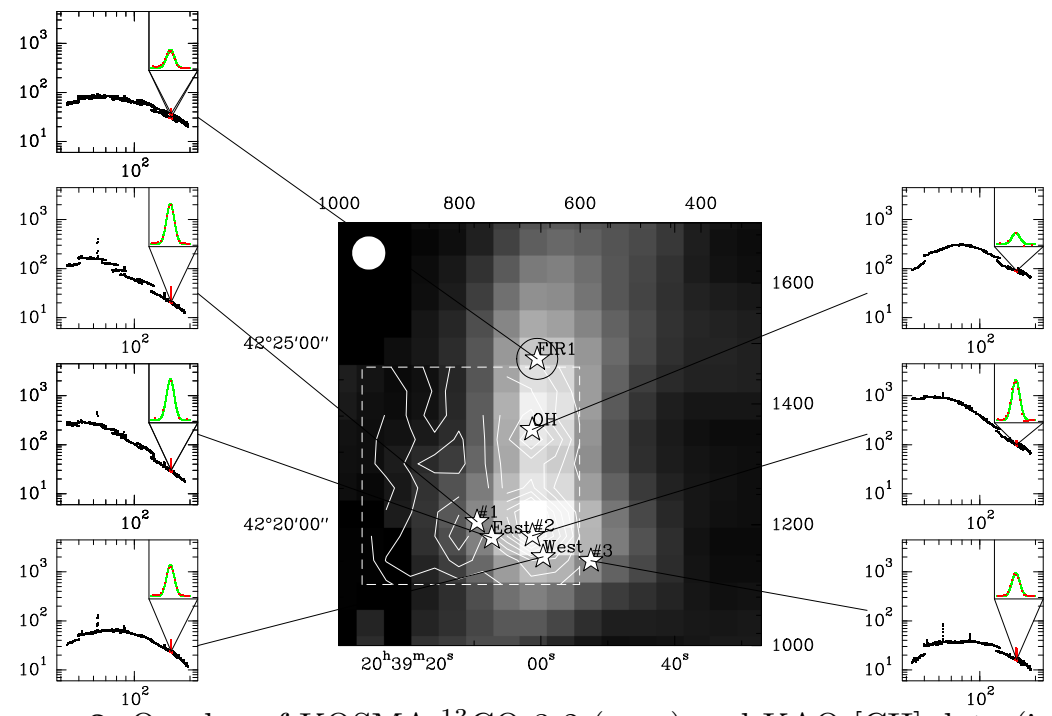

Figure 3: Overlay of KOSMA ${ }^{13} \mathrm{CO} 3-2$ (gray) and KAO [CII] data (in contours). The boxes show the ISO/LWS-FIR continuum and lines (e.g. high-J CO, [CII], [OI] and [OIII]) with a closeup view on the (unresolved) [CII] line at $158 \mu \mathrm{m}$ on the right. Circles indicate the KOSMA and ISO beamwidths.

At position $\# 2$, the continuum peak corresponds to $\mathrm{L}_{44-197 \mu \mathrm{m}}=4 \times$ $10^{4} \mathrm{~L}_{\odot}$. This result is consistent with earlier results (Harvey et al. 1977), when using the old distance estimate of $3 \mathrm{kpc}$ instead of $1.5 \mathrm{kpc}$. All major cooling lines are most pronounced, but the line cooling efficiency $\left(\mathrm{L}_{\text {Lines }} / \mathrm{L}_{\text {Cont }}\right)$ is much lower than towards the eastern and western lobes (up to $1 \%$ at position $\# 1$ ). Especially to the east, the $63 \mu \mathrm{m}[\mathrm{OI}]$ line intensity shows a strong eastwest asymmetry and extends beyond the continuum source.

\section{Molecular Gas: Physical Conditions}

We compare the observed ${ }^{12} \mathrm{CO}$ and ${ }^{13} \mathrm{CO}$ line intensities for position \#2 with a set of escape probability models at different $\mathrm{CO}$ column and $\mathrm{H}_{2}$ volume 
densities, as well as kinetic temperatures. Two models are found to match the KOSMA and ISO line intensities (see Figure 4). However, $\mathrm{n}\left(\mathrm{H}_{2}\right)$ varies by a factor of 10 between the two solutions, whereas $\mathrm{N}(\mathrm{CO})$ and $\mathrm{T}_{\text {kin }}$ are better constrained. We therefore included a ${ }^{12} \mathrm{CO}$ and ${ }^{13} \mathrm{CO} 9-8$ observation of comparable beam width (Boreiko \& Betz 1991). It is compatible with the $10^{4} \mathrm{~cm}^{-3}$ model, but since ${ }^{12} \mathrm{CO} 9-8$ is not fitting at all, we presume a calibration error for both lines. More valuable ${ }^{13} \mathrm{CO}$ observations with high-J are planed with KOSMA.

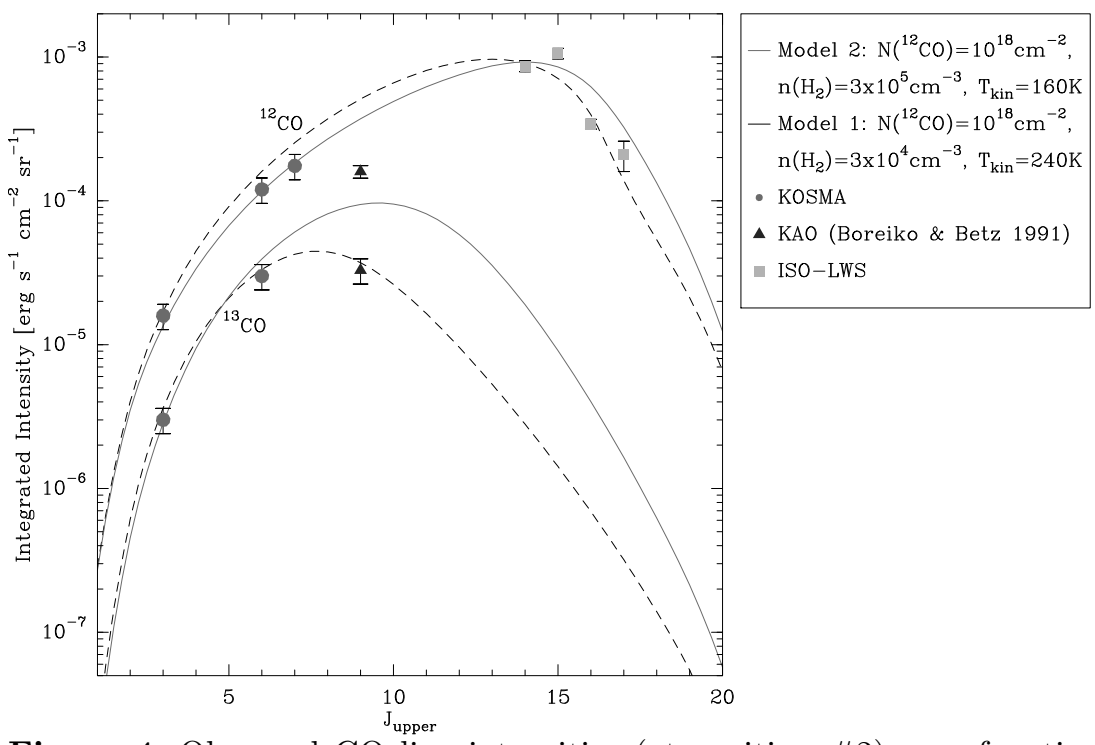

Figure 4: Observed $\mathrm{CO}$ line intensities (at position \#2) as a function of $J_{\text {upper }}$. The two lines represent best-fitting escape probability models assuming a ${ }^{12} \mathrm{CO} /{ }^{13} \mathrm{CO}$ abundance of 33 . The strong self absorption dip seen in the low-J and mid-J CO lines has not been accounted for.

\section{Perspectives}

With this study, we aim at extending our observations of the emission due to the major coolants of PDRs in Galactic star forming regions (e.g. S106 Schneider et al. 2003; W3 Kramer et al., in prep.). These data constitute the basis for the application of radiative transfer and chemical PDR models in order to 1. constrain the physical conditions in regions of high mass star formation and 2. show whether all important physical processes are taken into account in the current models to reproduce the observed line intensities and ratios. Also, it allows to derive a consistent picture of the photon dominated regions in the vicinity of massive star formation. This serves as a preparatory work for future missions like SOFIA and Herschel, which will provide a wealth of new information including velocity resolved [CII] data. 